\title{
What is the Experience of Receiving Health Care for Neck Pain?
}

\author{
Joy C. MacDermid ${ }^{*}$, , David M. Walton ${ }^{2}$, Jordan Miller $^{3}$ and $\mathrm{ICON}^{\S}$ \\ ${ }^{I}$ School of Rehabilitation Science, McMaster University, Hamilton, Ontario and Clinical Research Lab, Hand and \\ Upper Limb Centre, St. Joseph's Health Centre, London, Ontario, Canada \\ ${ }^{2}$ School of Physical Therapy, Western University, London, Ontario, Canada \\ ${ }^{3}$ School of Rehabilitation Science, McMaster University, Hamilton, Ontario, Canada
}

\begin{abstract}
This study used a descriptive phenomenological approach to describe the experience of finding and receiving health services for neck pain. Nineteen participants (18 females, 1 male) with neck pain (>3 months) were interviewed using a semi-structured questionnaire guide. Interviews were recorded, transcribed and coded. Two overarching themes described the experience: complexity in finding effective health care; and the need for informative, personalized, respectful communication. Complexity in finding effective health care was attributed to the variable approach and effectiveness of different health professionals, the need to experiment with care to find what works, the need to differentiate temporary versus permanent treatment effects, concerns about treatment side effects and the sense that financial factors influence personal treatment choices and provider behaviours. The need for informative, personalized, respectful communications was broken down into the following subthemes: the importance of being listened to, seen and believed; the need for useful information; and a desire to have outcomes formally tracked as a means of individualizing treatment. Overall, patients struggled to navigate the variable health services and providers that were available and that provided variable outcomes. They often did so through a trial and error approach. As such, patients remain open to unproven, even controversial treatment options. Research evidence was not a key ingredient in patient decision-making about accessing health services. The environmental, personal, health behavior factors interacted to contribute to health service utilization and would increase the burden of these for both the individual and society at large. The effectiveness of neck pain interventions is dependent on complex interactions between the context, individual, and health care provider, therefore, physiological responses cannot be considered as being distinct from these determinants.
\end{abstract}

Keywords: Neck pain, health service, effectiveness, utilization, qualitative, communication, access.

\section{INTRODUCTION}

Neck pain is the third most common area affected by musculoskeletal pain [1], with a third of all adults experiencing neck pain each year [2]. The course of neck pain is often episodic [3-6], and a proportion develops into chronic symptoms that are associated with disability, work loss and health care costs [7, 8]. Approximately half of chronic spinal pain is characterized as being intense; $40 \%$ as disabling, and $20 \%$ as intense, disabling, and chronic [9]. Given that neck pain is common, has high initial intensity and a substantial portion of chronic disablement, it is important to understand health service delivery aimed at reducing this burden.

The overviews of evidence on neck pain by our research team have indicated that a broad array of interventions are used in managing neck pain and have been evaluated in randomized control trials The most consistently used intervention would be considered "conservative management".

*Address correspondence to this author at the School of Rehabilitation Science, IAHS, 1400 Main Street West, 4th Floor, Hamilton, ON, L8S 1C7, Canada; Tel: 905-525-9140, Ext. 22524; Fax: 905-524-0069;

E-mail: macderj@mcmaster.ca

${ }^{8}$ International Collaboration on Neck (ICON).
We identified interventions that provide moderate relief of pain and disability. However, there is wide diversity in the types of interventions, providers and dosage that were used within current studies.

Neck pain causes people to seek a variety of health services, and these contribute to health care costs which can be difficult to track in most health care systems since they occur across multiple layers of the system. In the Netherlands, it was possible to glean a relatively thorough accounting of these by using data from national registries, reports of research institutes and health care authorities. The total cost of neck pain in the Netherlands was estimated to be about $1 \%$ of all health care expenditures and $0.1 \%$ of the country's Gross Domestic Product. About a quarter of the direct costs were for "paramedical" care [10]. These services include a variety of health care professionals such as physical therapists, chiropractors, massage therapists and others.

General practitioners (GPs), physical therapists (PTs) and chiropractors are the most common providers who manage neck pain. One study found that in the United States, $61 \%$ of patients received primary neck care from medical doctors, $28 \%$ received care from chiropractors, and $11 \%$ received care from both a medical doctor and physiotherapist [11]. Given the range of options/practitioners that can be used to manage neck pain, there is a rationale to investigate different 
health service delivery options from a cost-effectiveness perspective. A recent systematic review of this issue [12] found five economic evaluations based on randomised controlled trials that included a cost-utility and/or effectiveness analysis. Studies found conflicting results on different combinations of conservative management. Heterogeneity existed between the studies on participants, interventions, controls, outcomes, follow-up duration and the study context. As a result, the systematic review was unable to make firm conclusions about the cost effectiveness of different approaches to manage neck pain.

Quantitative research provides an important perspective on the costs and effectiveness of health services; but qualitative research can be important to provide a richer understanding of the experience of receiving health care for neck pain. Existing qualitative studies suggest that there are differences in perspective and priorities between providers and patients that might contribute to dissatisfaction. GPs have expressed concern about the costs of neck pain treatment suggesting that their patients expect definitive and expensive forms of therapy, while they prefer cost-efficient forms of therapy $[13,14]$. They felt that effective interventions such as promoting physical activity and having patients acknowledge and address the psychological component to their neck pain were beneficial; but not well received by patients. This disconnect was confirmed in another study of patient-general practitioner pairs where patients and their health care provider expressed discordant perceptions on psychological distress following whiplash injury [15].

Qualitative studies that have focused on the patients' perspectives confirm discordant perceptions. Patients seeking health care services acknowledge that they do seek interventions that might relieve their symptoms, including massage, physiotherapy or injections [16]. However, they also reported attempts to enact self-management; and that they consulted their family practitioner only when these selfhelp strategies failed. The study suggested that the interaction between doctor and patient was "distant, contributing to both sides avoiding issues that might touch upon psychological aspects of neck pain" [16]. Our recent descriptive study that defined the experience of neck pain demonstrated that a considerable proportion of patients readily acknowledge that stress aggravates their symptoms (submitted paper). We suggested that patients may react more positively to health care interactions where psychosocial components are treated as an irritant to their symptoms, rather than ones where it is provided as an explanation.

Qualitative studies to date have focused on the patientphysician dynamic and largely ignored other providers.
Therefore, the purpose of this study was to describe the individual's experience with receiving health services for neck pain without reference to specific providers, in order to allow patients to reflect on their own personal experiences across different providers.

\section{METHODS}

\section{Research Approach: Descriptive Phenomenology}

Phenomenological studies such as ours aim to describe individuals' experiences and interpretations of a particular phenomenon/experience. The primary purpose of this study was to describe the experience of receiving health care for neck pain and to determine the meaning of that experience for individuals living with neck pain.

\section{Participant Recruitment and Description}

Sampling was conducted to include a diverse group of people with neck pain. Exclusions were used for people whose health problems might predominate their neck pain; or who were unable to communicate effectively about their neck pain. The study was conducted at two sites: Canada and Australia based on the research team's location during the study. Participants were recruited from flyers posted on the university campus, web-based advertisements and a database of research subjects with neck pain who volunteered to be contacted for additional research opportunities. Inclusion criteria were: 1) 18 years of age and older; 2) had neck pain with or without arm/shoulder pain, chronic or recurrent (recurrent being more than one episode in the past 3 months). Patients who reported pain at the posterior aspect of their neck were included, regardless of whether this pain was also associated with more diffuse neck pain patterns that included the following: headache, shoulder pain, numbness/ tingling in the hand/arm.

\section{Collection of Data on Participant Experiences}

To identify the care experience, 19 semi-structured, faceto face or telephone interviews were conducted and recorded for purpose of analysis. Sixteen interviews were conducted in Canada; 3 interviews were conducted in Australia (based on location of the investigator). Sampling continued until data saturation was confirmed, including confirmation that the three interviews in Australia provided similar findings to Canadian respondents. Telephone interviewing was used when preferred by participants and has been shown to be a reliable and efficient method of obtaining individual experiences when compared to face-to-face interviews [17]. Three independent researchers conducted the interviews lasting approximately 45 minutes. All questions were vetted by an expert committee and trialed on practice respondents prior to initiation of the study. Calibration of questioning was discussed by interviewers.

\begin{tabular}{|c|c|c|}
\hline Demographic & Canada $(n=16)$ & Australia $(\mathrm{n}=3)$ \\
\hline Gender & 15 females, 1 male & 3 females \\
\hline Pain intensity ( $0=$ no pain, $10=$ worst pain imaginable $)$ & $5.2($ range $=2-9 ; \mathrm{SD}=2.2)$ & Not available \\
\hline \# Participants with history of whiplash injury & 3 & 3 \\
\hline
\end{tabular}


Semi-structured interviews were conducted using a questionnaire guide that was framed to identify what care patients had received, and their experiences with accessing effective health service. The interview questions started with broad open questions that were intended to set an open, unbiased, receptive, presence. "What care have you received for your neck pain?"; "Can you tell me more about that?"; "How did that work for you?" Probing questions were used to delve further into themes that emerged through the open questioning, starting with broader probes, e.g., "Can you tell me more about that?"; moving to more specific probes based on the experience brought forward by the participant, e.g., "Were you included in making decisions?" (when decisionmaking was raised), "What information did you need?" (when a communication theme was raised).

\section{Managing, Organizing and Analyzing Data}

Data management included audio-taping and verbatim transcription of interviews. Electronic audiotapes and texts were provided to the analysis team (JM, DW). The transcribed interviews were reviewed by two researchers who independently coded the interview transcripts. The analysis followed six phases of analysis: familiarizing with data, generating initial codes, searching for themes, reviewing and organizing themes, defining and naming themes, and producing the report. Coding of text was performed by an initial reading of manuscripts for overall meaning, followed by coding of individual text segments into their meaning. Coding was performed using a modified Stevick-Colaizzi-Keen [18, 19] where individual textural and structural descriptions were developed; followed by composite textural and structural descriptions, and then a synthesis of textural and structural meanings and essences of the described experiences. The first order (respondent stated) constructs were grouped and classified into common meaning groups. The overall essence or meaning of these second order (researcher derived) constructs were interpreted by investigators. The "essence" of the experience illustrated by respondents was organized by themes. Themes were organized into two overarching themes. The researchers arbitrated any disagreement with coding/themes, any bracketed assumptions, and agreed on the subset of quotes that reflected the themes.

\section{Trustworthiness of the Data/Findings}

To verify the trustworthiness of the data, the identified themes and quotes were also reviewed with the study participants ("member checking"). Feedback was requested, including identification of themes that might have been missed, and whether those described were consistent with their intended expressions of experience. Participants agreed with all of the summarized findings. The draft manuscript was also reviewed by clinicians and individuals with neck pain outside of the study respondents who commented on the organization/writing of the manuscript and provided feedback on whether it reflected themes consistent with their own experiences and understanding of qualitative research. This was used to enhance the clarity of the manuscript, but not to change any of the themes derived from participants.

\section{Findings}

Based on review and analysis of the participant responses, 8 themes were identified and these were organized in two overarching themes (Fig. 1). The two overarching themes were: 1. complexity in finding effective health care; and 2. need for informative, personalized, respectful communication. All study participants provided agreement with the identified themes. A description of each of the identified themes with associated quotes is presented.

\section{THEMES}

\section{Complexity in Finding Effective Health Care}

1. Variable Approach and Effectiveness of Different Health Professionals

2. The Need to Experiment with Care to Find What Works

3. Temporary Versus Permanent Treatment

4. Concerns about Treatment Side Effects

5. Financial Factors Influence Treatment Choices and Provider Behaviors

\section{Need for Informative, Personalized, Respectful Communications}

6. Importance of Being Listened to, Seen and Believed.

7. The Need For Useful Information

8. A Desire to have Outcomes Tracked

Fig. (1). Identified themes.

\section{Complexity in Finding Effective Health Care}

The first and most predominant overarching theme was the complexity patients experience in finding health care services that met their needs for managing neck pain. Complexity existed along several axes. Taken together, the described complex experiences resulted in substantial challenge for patients navigating health services for their neck pain symptoms.

Variable Approach and Effectiveness of Different Health Professionals

One of the most predominant themes mentioned by all respondents was the variability in approach and effects they experienced at the hands of different health care providers. Many participants had an experience where receiving a specific health service from a provider had not helped them (or had worsened their symptoms). In some cases, this was expressed as having,

\section{"been there, done that" ... "I have books and things like that; on pain medication and Tai Chi. I have all that stuff at home. Tried it, didn't like it ... or didn't work."}

In other cases, respondents were more distressed because they felt that the health service had actually increased their symptoms. It was often difficult for participants to be certain whether it was the health service or some other factor that caused this worsening, but worsening of symptoms while receiving treatment led patients to believe that the specific health care provider was responsible. Conversely, a few participants articulated an experience where they could differentiate that a health service was effective for specific subcomponents of their neck pain problem, but not for others. In some cases, they were able to relate this to a 
specific symptom or physical impairment that responded to certain interventions.

\begin{abstract}
"The only time that the chiropractor actually did help me was when the neck would not move from side to side". or
\end{abstract}

"I even had acupuncture. It works really well for migraines, but it didn't seem to do much for my other aches and pains."

However, it was more common that participants attributed the variability in effectiveness to the providers, rather than any aspect of their health problem or their personal characteristics. Participants had clearly experienced variability with treatment effectiveness even within the same profession.

"I've had acupuncture from a couple of different people, and one person, when they did it, it worked and the other person, it was terrible. I've also tried chiropractic treatment. That has worked on certain occasions, and on others, it hasn't. And physiotherapy, I truly believe it's the person doing the physiotherapy that makes the big difference rather than the treatment."

One dissenting viewpoint was expressed by a participant who suggested that they had experienced a consistent approach across one profession, although it was not clear if this consistency was necessarily valued.

"Physios usually do some hands on manipulation of the neck and give you exercises; it is pretty regular. All physiotherapists have the same set of exercises that you do for the neck. I went to three and they are pretty similar."

It was evident that the participants' experiences and expectations of variable outcomes at the hands of different providers evolved over time. For some individuals, this realization contributed to their sense of needing to take personal responsibility to find the person or service that worked for them. For others, it was more apparent that this negatively impacted the trust relationship between patients and their health care providers.

"I ended up in this situation in the medical profession and also the wide health care arena, where I had to trust that what every individual was telling me was the correct thing for me. But then I realized that actually they all just have their own tool kits of treatments and therapies, and that's what they're pushing...”

Since the purpose of this study was to explore the experience of receiving health care services, not the experience of operationalizing self-management, we did not explore strategies or limitations in conducting selfmanagement. Despite a lack of probing on this issue, a subset of the participants volunteered that self-management was fundamental to their health and interpreted this as being more independent from health care providers.
"You see, some of the basic things that really work are swimming and walking, believe it or not, rather than seeing someone."

The Need to Experiment with Care to Find What Works

The variable responses that participants had experienced with the effectiveness of the health care may have contributed to the next theme-the need to be able to experiment with different treatments and providers to find what worked. Almost all participants reported experimenting with different treatments to find what worked, and the majority demonstrated openness to a wide variety of options. Only two participants expressed concerns with the scientific basis for treatment. These patients were concerned about the "pseudo-medical" treatments-terminology that they used to describe complementary or alternative therapies.

Conversely, other participants expressed frustration that they were not able to access services that they wished to "try" and appeared to be open to many alternatives, including a number of interventions where there is no evidence supporting effectiveness. Some participants had seen advertisements for "new" treatments for spinal pain; but felt that they were being denied access to these treatments because they were "not covered" under their health care benefits.

Participants conveyed their need to be open to experimentation if they were to find the health services that would work for them. This was illustrated by a participant who stated:

"Yes and I am willing to try anything, and I have tried everything that I could think of; from bone therapy to osteopathy to three physiotherapists. Everything that I think is available, I have tried. The ones that worked for me in the end was physiotherapy. But there's a qualifier on that, you've got to find the right physiotherapist ... I've tried probably about 3 physiotherapists, before I actually ended up in the right hands."

Other participants echoed this experience and reflected that searching for effective care is an ongoing experience. For people living with chronic neck pain, the ongoing search to access health professional services that are effective in relieving their symptoms becomes embedded in their ongoing process for managing their neck pain.

"I've tried a lot of treatments over the past, almost 15 years now. I've been to some physiotherapists who are fantastic and others who I don't think believe you."

A number of participants appeared to be comfortable with experimentation to find the right provider as if they understood that it was inherent in dealing with their neck pain. Others reflected frustration or a lack of trust of health professionals because of the variability in health care providers.

"I had to go through 3 years of terrible interactions. See, I trusted everybody. I trusted them because they had their degrees and were 
medically qualified that they would be OK, but no..."

Although the symptoms and treatment responses were quite variable across participants, it was notable that participants attributed their variation in outcomes to the provider, not the characteristics of their neck pain or health behaviors. Despite this, it was clear that patients' experiences could be widely divergent to the same intervention. For example, the majority of participants who had massage therapy noted that it was pleasant and provided temporary relief of symptoms, although a less typical response was noted by one:

“(I) can't take any massage. I haven't tried it for 2 years. When I had massage... the first time I ended up in the hospital going off in an ambulance because it brought on severe sternum pain."

Some patients felt that their doctors were not supportive of some of the "experimenting" they were doing, in particular, if it involved complementary or alternative medicines. Some described their family physician as dismissive of options outside of the traditional medical realm, as illustrated by this quote:

"I don't think that doctors are in tune with natural products as much as I would like. Like, if I'd mention that I went to a naturopath; I actually had the doctor roll his eyes. So they are not always into complementary medicine, I would like to see it more from health care providers."

When the participants were discussing their experiences with GPs, it was apparent that the amount of time physicians were able to allocate to them, the degree of interest expressed and the apparent knowledge of their physician about neck pain all contributed to whether they felt that their family physician was motivated and competent to help them with their neck pain problem.

"So the first thing you do is go see a GP, and that's where the problem starts because they don't have the time, they don't have the knowledge, and they don't have the interest. So they need to be briefed on what do you do with a person coming in with neck pain. They don't seem to know, so all they want to do is send you to a rehab specialist or a physio, or give you opiates to help with the pain."

\section{Temporary Versus Permanent Treatment Outcomes}

One aspect of care that clearly increased the complexity of seeking effective health care was that some treatments provided short term relief or "felt good" but did not contribute to sustained or long-term improvement in their neck disorder. Massage was commonly mentioned as one of these types of interventions.

"I really enjoyed the massage and heat treatment. That was great. At the time it was wonderful, but it never really did the job."

Conversely, a number of participants seemed to be aware that some of the more "difficult" interventions were the ones that resulted in the most long-term benefit. In some cases, patients even reflected that short-term inconvenience was an inherent component of achieving this longer-term benefit.

"I don't enjoy doing the exercises, but I know I have to do them and I see results, so I do them."

It was evident that a number of participants had learned to discriminate between the short-term and long-term effects. This was particularly challenging in cases where these interventions had temporary adverse effects or required more active participation.

\section{"The physio can be painful because I am being moved into positions that I am not used to. They are the most beneficial though ... by far..."}

Exercise was the most commonly mentioned intervention that was perceived as challenging, but effective. Participants often noted that exercise interventions require more effort on their part and while they valued the longer-term benefits of exercise, they also acknowledged challenges in maintaining adherence.
"You tend to try and come up with all these things that you really should be doing rather than the exercises, but at the end of the day, the exercises, I guilt myself into it because I see results and I know I have to do them."

\section{Concerns About Treatment Side Effects}

A number of participants were concerned about safety and side effects of different interventions. The most common concern was side effects that can occur from medications for neck pain. Some participants acknowledged that medications were necessary or helpful at some time points, but they preferred to avoid them. Although side effects were the most commonly expressed concern, those who mentioned it often assumed that others would not necessarily share their concern.

\section{"I've taken pain killers for it before and I prefer not to have to take pills, but that's just me ... really hesitant towards medication."}

A number of participants had legitimate reasons to be concerned about adverse side effects with medications as they had personal experience with an adverse event or side effect. The most common concern was the effect of medications on cognitive function.

"Well I would try it for a short time, and if it worked, fine I'd be as happy as could be. But most of the time, especially with magic pills, you end up being a bit "zoo-ed" and not all there. You walk around like a zombie. I like clarity; I don't want to be walking around like a stoner."

or as stated by another:
"[Drug name withheld] - terrible experience that took months from my life ... It messes with your brain."

A number of the people who had taken medications discussed the challenge of making personal risk-benefit 
decisions. While the majority acknowledged that they had experienced some relief of symptoms, they also felt conflicted about the side effects. Therefore, a number of participants spoke about undergoing a concerted effort to become "free" from medications. Given this, the need to return to taking medications was seen as worsening of their condition.

"I used to take a lot of medications so I am trying to get everything out of my system ... I tried for a year ... I stopped taking Advil, but I just couldn't deal with this summer."

Although medication was clearly the most predominant area of concern with respect to side effects, it was not the only intervention where participants had experienced an adverse event. For example, one participant experienced an unusual response to acupuncture that would be considered an adverse event.

\section{"One time I had acupuncture in the muscle that goes up between the neck and the shoulder this big muscle here and it all swelled up. So I really didn't enjoy that and it made me a little leery; but I know that acupuncture can be really good for you and it is supposed to be really good. I haven't had it since because of the swelling reaction that I had."}

Other respondents had not experienced a side effect/adverse event but had changed their use of health services because of their perceptions of risk for an adverse event. The most common concern in this regard was for chiropractic treatment (manipulation). Participants who reflected this concern changed their behaviour on use of this health service based on recently acquired information, although they were not always clear about the source of this information.
"Well I went to a chiropractor for a long time, but he used to snap my neck for a long time. I stopped letting him do that, because that's when it first came out that if you do that, sometimes you can have a stroke. I was a wee bit concerned about the stroke thing, so I said "let's just concentrate on something else." ... I didn't exactly want to be walking around gimpy."

Finances Influence Treatment Seeking and Provider Behaviours

Financial influences clearly affected the choice and overall use of health services. Participants reflected that financial concerns (either theirs or the providers) could impact on what health services were offered or accessed. Some participants reported that their personal treatment choices were affected by the extent to which treatments were covered by their health benefits. Others expressed concern that financial considerations may be motivating the treatments that they are offered.

\footnotetext{
"A lot of things are not covered by the benefits that are out there now. The cost is sometimes a factor that you can't take some of the treatments that you see advertised like that spinal decompression ... and you just can't
}

afford it ... so I wouldn't know if it works or doesn't because it is too expensive."

Some participants, particularly those who had pursued multiple treatments over time, were frustrated by the overall financial impact on themselves and some expressed a sense that money may be a driver in the system.

"The whole process is very money-hungry ...
I've spent an absolute fortune on trying all
these different things."

Need For Informative, Personalized, Respectful Communication

The second overarching theme was the need for informative, personalized and respectful communication. Communication clearly contributed to the trust relationship with health care professionals, and the effectiveness of interventions provided by different health care professionals. In addition, participants wanted communication that went beyond empathy to also include "useful" information. Subthemes below indicate aspects of the dynamic communication issues.

\section{Importance of Being Listened To, Seen and Believed}

A theme across the majority of participants was the need to be listened to and have their condition acknowledged as legitimate. In the absence of definitive tests that can prove patients with neck pain have a real health condition, many find that having their health care provider believe that their problem was real, including their truthfulness in reporting its fluctuations, was fundamental to establishing an effective working relationship. Similarly, several participants described the importance of their health care provider understanding the unique elements of their neck condition. Participants wanted their health care provider to understand their struggles in dealing with the fluctuations of their neck pain and the inconsistency of their symptoms over time.

Participants also wanted their health care provider to see how difficult it could be to live with neck pain. A few participants expressed the concern that their condition was not really of interest to others, suggesting that some practitioners found their condition "boring" or that they were "not interested". A common theme across participants was the challenge in getting their GP to appreciate their neck pain. However, it was clearly important to participants that this happened. Some participants described experiences of being "talked over" or being the recipient of skepticism from their provider. This dismissive experience was perceived as a lack of respect and interfered with the therapeutic relationship in a way that was a barrier to effective treatment. Some participants expressed that their interpretation of the quality of the clinician was related to the degree to which they acknowledged the legitimacy of the symptoms and challenges of living with neck pain.

“... (Important) that the doctor listens to me and acknowledges that there is pain because my doctor right now, when I first went to her when I was like 12, she said that I was crazy because I was too young to have neck and back pain. It's hard to find a good doctor that pays attention to what the problem is..." 
It was important to participants that they felt that their health care provider saw them as trustworthy individuals, recognizing and respecting their personal circumstances and how their neck pain impacted their personal lives. Participants interpreted being seen as individuals through the nature of their interactions with health care providers. When dealing specifically with GPs, the dynamics of the relationship were often based on verbal interactions. Participants found these interactions rewarding if they felt listened to, and saw signs that their condition was acknowledged.

(Negative interaction) "My physician
suggested that I stop using the computer...
But she knew I was a PhD student."-- versus
"she knows I need to deal with my son and
always asks about it." (Positive interaction)

The experience of being patient centered extended beyond verbal interactions. Participants also focused on observations and actions that providers undertook within the clinical interaction. Physiotherapists who were able to customize treatment in ways that met the individual's needs, or who shared/acted on observations conveyed that they were "seeing" the participant. These overt behaviors were valued by participants as they were concrete indicators of being responsive to the individual's needs.

\begin{abstract}
"Just the way he actually takes an interest in me and what's going on. And he always asks me about my work and how that's going. You've got to be able to feel comfortable and trust the person. ... shows that he's not just "do this, do that, and off you go", out the door and I'll see you next week. He takes the time to review the new exercise that he gave me the day before and "let me see again how you're doing this one" and "how are you finding the neck? Are you driving alright?" He asks about the headaches."
\end{abstract}

One participant described an experience where her provider became 'excited' at an improvement in function. The participant felt that since her therapist was paying enough attention to her that she could notice this small advance in her ability to do something, then she must be paying close personal attention to her progress. Further, the fact that the provider demonstrated excitement gave this participant a sense that the provider was truly invested in her care and health outcomes.

"He (my physiotherapist) was excited about me looking at the ceiling the other day."

Some participants noted that the feeling of being understood extended beyond health care providers and included the office staff.

“They are very pleasant in the doctor's office. They continually ask, how is the pain. They ask me how I manage my work life and at home, how am I dealing with everyday tasks. That's helpful."

Other participants felt depersonalized by certain health care providers and were unable to establish a collaborative relationship.

\begin{abstract}
"The one doctor is really good. The other one I was just a puppet for her. She was trying medication on me and she kept on switching and switching and she would write it down and was really excited about it and I was like "hello I am not your guinea pig." And I got really pissed off and I just stopped everything."
\end{abstract}

Despite the importance of a healthy therapeutic relationship, only one participant mentioned an experience where health professionals actually asked for feedback on the nature of this interaction. When this occurred, it was valued and contributed to a positive relationship.

\section{“... she actually came out and asked me if I was satisfied with the options we were coming up with. She is very good and open to work that way."}

In some cases, participants expressed empathy towards their health care providers, recognizing that neck pain might not be the most exciting issue to treat. This was particularly true for GPs, where participants had interpreted that their family physicians' lack of interest might be valid since other people might have more interesting or pressing health problems. However, other participants described changing health providers because they felt that the interpersonal interactions were not satisfactory. An unsatisfactory primary health care relationship was evident where participants experienced a lack of understanding of their neck pain problem, or felt that it was not being prioritized as important by their provider. Failure to achieve relief of symptoms contributed to an unsatisfactory relationship across all provider types. Some participants had sought different family physicians because of these frustrations and were able to find a different treatment experience. In other cases, the participants simply encountered new barriers to effective treatment when interacting with their new provider.

\section{"I changed G.P. and immediately I knew that he was going to help me because he was listening -I booked a double appointment, I thought that was the first thing I needed to do so he wasn't going to shoo me out of his office in a hurry... and he listened."}

Some participants were reluctant to change their primary health care provider even though they were not satisfied with how their neck pain was managed because they reflected upon their overall interactions with their family physician across different health problems and had found them to be better at managing other disorders. Others were reluctant to change providers because of logistic challenges in acquiring a new health provider. Where participants had decided they did not want to change providers, they focused on developing a strategy for communicating more effectively with their provider by adopting techniques to get more attention to their problem such as booking a double appointment or coming with a prepared "story".

\section{The Need For Useful Information}

Although participants expressed a strong need for empathy in the patient-health care provider relationship, it was evident that empathy alone was not sufficient for the 
relationship to be seen as productive. Participants expressed the need for useful/practical information. In addition to being empathetic, patients wanted health care providers to initiate strategies that resulted in meaningful change in their condition. Without this, the health care provider was perceived as being nice, but ineffective.

\begin{abstract}
"She just wanted to talk. She'd just sit and she would be like "tell me about your life and tell me what is going on" and when I would say things she would be "you know that's why you are stressed because of this and that and you know it is hard" and she would be really concerned and comforting ... but she didn't give me any solutions."
\end{abstract}

Participants reported the need for additional information on their neck problem and also expressed concerns about the quality of the information received. Some participants expressed frustration that the information provided was not useful. This frustration was specifically mentioned in relation to diagnostic and treatment information. Patients expressed frustration when no one was able to provide an explanation for their symptoms and that there was no clear direction on how to resolve it.

Diagnostic tests were often experienced as being counterproductive in this regard. Participants expressed a pre-test hopefulness that a diagnostic test would resolve the uncertainty about their neck pain. However, it was more common that diagnostic tests "did not show anything". Participants perceived that their health care provider was happy to see that there was nothing seriously wrong on the diagnostic test. However for them, this was experienced as continued inability to explain their pain and a further lack of validation of their problem. Rather than feeling relieved when no serious pathology was discovered, a number of participants found the lack of validation to be a stressor.

In addition, participants were frustrated that their providers could not provide any practical information that could be applied to their daily lives. Both were reflected in this quote:

\section{"I remember I went for my MRIs they would say that it is all in my head and I would get pissed off ... they are like "just relax, take it easy" I am like "no I want more information about what I should do, what would benefit me?"}

One specific area in which a number of patients felt they needed more information was about the treatments they were being offered. Participants wanted to know how the treatments worked and what they should expect.

\section{"I would prefer more information about each treatment. I know when I go in they give me the neck treatments and I go out and I am feeling better but I don't know what is happening with my body."}

Several patients expressed a need for more informed participation in their decision-making around treatment. It was not enough to know about the treatments but they wanted to be actively involved in making the selection between different treatment options. It was clear they felt they needed more information to be able to do so.

\section{"Well first they should explain the different \\ types of treatments and then let me choose."}

Several participants mentioned the need for concrete informational resources. Content specifically mentioned for these resources included exercise programs, methods for managing their neck pain, coping strategies and information about their activity levels and restrictions. It was clear that patients valued "take away" information that could be used outside of treatment sessions.

\section{"I think that they should probably give out handouts or at least give a chart with exercises."}

\section{A Desire to have Outcomes Tracked}

The complexity of navigating effective health care services presents challenges for those living with neck pain. Several participants were specific about the need for ongoing measurement of their status as feedback that would help them and their providers arbitrate the effects of their health care. Some expressed a belief that health care providers would be better able to optimize their health care if they had more structured evaluations. These assessments were viewed as being necessary because of the variability in their symptoms and abilities on a day-to-day basis. Participants believed that it was important for clinicians to understand the nature and extent of these fluctuations; and to match their treatments to their unique and changing status. Participants reflected that this information was required for them to adapt their physical activity to the fluctuations in their symptoms and abilities. Patients also expressed that ongoing monitoring was important for motivation or adherence to certain treatments such as exercise.

"Maybe more assessments, ongoing assessments
would be helpful because I found that my neck
pain changes constantly ... so I can track my
progress."

\section{DISCUSSION}

This study evaluated the experience of receiving care for neck pain and found two primary themes: complexity in finding effective health care, and need for informative, personalized, respectful communication. Complexity was increased because of the variability in the experience of neck pain, the wide range of treatment options with variable effects, and the fact that the same intervention could provide very different outcomes with different providers. In fact, participants came to realize that effective health services were very dependent on the provider. Given that neck pain is often managed conservatively, these provider effects and communication issues must be considered an important element of treatment.

The overviews in this issue have indicated that there is a subset of effective interventions that produce a mean small to moderately-sized treatment effects in relief of neck pain and disability. However, what is underappreciated by quantitative syntheses is that effectiveness potential can be dissipated within clinical trials by the lack of consistent effects across providers. This variation was noticed by our participants within professional disciplines, so was not 
directly attributable to professional training. This supports the need to attend to provider effects during design and analysis of clinical trials [20].

In some senses, individual clinicians treating neck pain might be better considered as a "complex intervention" which are defined as interventions "made up of various interconnecting parts" [21] since treatment is often multimodal; and multiple issues will impact on the effectiveness potential of a given treatment approach/provider. Interventions that are delivered directly via the provider, such as massage, psychotherapy, chiropractic, surgery, and physiotherapy, all share the attribute that the provider and intervention are inseparable. The provider brings their individual skills, knowledge, beliefs, preferences and experiences into the administration of the intervention. These factors can independently or, in combination, modulate the effectiveness of a given treatment. This study also highlights that these provider effects will interact with patients' needs and preferences to affect treatment outcomes. Clinical research studies demand standardization to improve the internal validity of the study; and may have great difficulty dealing with this complexity where provider characteristics, patient characteristics, treatment characteristics, treatment combinations and the interactions between all of these might contribute to outcomes. This affects the external generalizability of trial results to individual clinicians or patients.

Neck pain studies rarely address how they attempted to limit or control these mediating variables in their research design [20]. Standardization within clinical practice or trials may be more achievable if it were possible to define the active ingredients in the therapeutic relationship. However, this is an elusive goal. Screening/certification of providers to a certain skill or training/calibration are approaches that may reduce provider variation in practice or research. Empathy, communication, and patient-centeredness skills may be more challenging to assess/standardize; but were clearly valued by participants in this study. Participants in this study and others $[22,23]$ clearly wanted to "be assessed and treated individually". Therefore, standardization implemented in clinical trials or practice (i.e., through clinical practice guidelines) must provide clinicians sufficient latitude to accomplish appropriate customization. Integration of quantitative and qualitative methods is encouraged in developing complex interventions before proceeding to full randomized control trials [21] and is clearly needed in neck pain trials.

It was evident that successful interaction included "being seen, and being on the same page" and that this contributed to the effectiveness of treatment. This is consistent with a systematic review [24] that suggests patient satisfaction and physical function improve to a greater extent in patients with musculoskeletal conditions when a strong alliance is formed between patients and their providers. This alliance was reflected in the participant themes around their need for informative, personalized, and respectful communication. Participants experienced greater conflict in their interactions with family physicians than other providers, and this is consistent with the findings of previous qualitative research that investigated these interactions and found discordance between perceptions and expectations [13-15].

It was clear that patients' judgments about the quality of their providers were primarily influenced by the communi- cation style and changes in symptoms. It was notable that being evidence-based, having advanced training/certification or more clinical experience were not mentioned by participants, suggesting these are not explicitly valued as a means of selecting a health care provider. In contrast, these factors are considered important when professionals judge competency.

Many participants had experienced health care services that were not helpful in reducing pain and disability. Some of these interventions were ones that did not have supporting evidence so the experience was not surprising. Rather than being cautious about trying treatments that were not evidence-based, participants appeared receptive even hopeful of trying as many interventions as needed to achieve relief of symptoms. This finding leads to questions about whether patients are receiving education on the evidence for different treatment options. Evidence does not appear to be part of the culture and experience and, thus, it is unclear if people with neck pain would embrace an evidence-based treatment approach. We know that there is evidence to refute some of the interventions being accessed. Therefore, with more information about the evidence, patients would need to deal with discordance between their current choices and the evidence. Conversely, some participants reported receiving no benefit from interventions that have moderate or high quality supporting evidence where moderate treatment relief should be expected. Within drug trials, patients have variable responses which are usually related to physiologic differences between individuals. The importance of provider effects would be an additive source of variable responses in neck disorders. It is clear patients require that their clinicians carefully monitor and individualize treatment based on their individual response.

Our findings are aligned with other qualitative studies [25] that suggest optimal communication is critical to success in management of neck pain. One of the strongest themes was the need to be listened to, understood and acknowledged. Previous research in the experience of seeking health care for neck pain has also identified the need to be understood [15]. Patients clearly need validation that their neck pain is "real" or, as cited by others, "to be taken seriously" [23]. If their health care provider does not convey this, then the sense of trust in the therapeutic relationship is disrupted $[26,27]$. Of note, in this study, imaging often negatively contributed to this dimension. Since imaging is often used to rule out serious problems, but rarely identifies a definitive problem, the patients' need for validation was worsened as a result of the diagnostic process. Similar concerns have been raised in low back pain [28].

Patients' experiences of high variation between providers may partially explain why patients and providers have different perceptions of the therapeutic relationship. GPs have expressed their roles as being stewards of the health care system [14] and are unlikely to recommend repeated use of a service that had not worked on a first exposure. However, they may not appreciate that the same service provided by a different person may have a very different outcome. Further, the very experience of inconsistent outcomes contributes to the patients' sense that experimentation with ineffective treatments is a necessary evil. 
The behavioral model of health services talks about access to care in terms of contextual characteristics, individual characteristics, health behaviors and outcomes $[29,30]$. Population-based health service access data indicate that neck pain patients see a mean of 5.2 provider types (mean of 21 visits) for neck pain [31]. The participants in this study were consistent with that behavior in that they had accessed multiple professions including medical, rehabilitative and complementary medicine. The traditional medical model which was characterized as "overly brief" family physician visits and use of medication did not appear suited to patients with neck pain. The majority of communication issues were with GPs. It was also perceived that family physicians did not have a positive attitude towards complementary medicines. Similar perceptions were noted by patients with respect to chiropractic treatment in a study of injured workers [32]. Patients' needs were more aligned with the rehabilitation profession's approach, but this avenue was sometimes contaminated by cost barriers. This would suggest a disconnect between health policy (contextual characteristics) and perceived needs (individual characteristics) since this work was conducted in a socialized medicine environment where visits to family physicians are fully insured, whereas rehabilitative services are not.

Health service data suggest that there is underutilization of effective treatments such as therapeutic exercise [31] in neck pain care. This was confirmed by patients' reports of being challenged to adhere to exercises they knew were effective. This is also consistent with the experience of physical therapists who experience a disconnect between their goal of empowering patients to exercise for selfmanagement of non-specific low back pain, and the patient's beliefs and attitudes [33]. These findings emphasize the importance of strategies to improve adherence to exercise in patients with neck pain. Previous studies have indicated that patients have concerns about time demands, complexity and effects of prescribed exercises. Other studies indicate that adherence is enhanced when health care providers present knowledge about the disease, promote feedback/motivation during exercise instruction, give reminders, or monitor results/adherence to exercises [13]. This is consistent with participants' interpretation that they were motivated to be more adherent to exercise if their therapists re-evaluated how they performed their exercises and measured outcomes. The link between outcome evaluations and adherence provides a concrete strategy for promoting adherence.

Variation in personal health services practices is a domain of the behavioral model of health service utilization. It is clear this domain is a substantial issue in neck pain health services. Participants in this study indicated they wanted more information about treatment options and to have a role in selecting from available options. This is consistent with the high satisfaction report when patient's preferences were used to direct referrals between a chiropractor, osteopath, or a physiotherapist for provision of manual therapy services for neck pain [34].

Medication was the intervention that caused the most concern for patients. A number had tried medications and acknowledged some relief of symptoms, but were concerned about the side effects. A number reported substantial cognitive deficits while on medication, and were unhappy with the extent to which these cognitive changes affected their lives. Several mentioned the struggle to become medication-free. A previous study of patients taking part in online discussion [35] found that patients discussed considerable mistrust of medications and medical practice. Discussion also described non-adherence and how these adherence issues are seldom discussed with health care providers [35]. The substantial side effects noted by participants in this study suggest that adherence may be related to adverse effects. Greater attention to adverse effects of medication is suggested by these findings.

Two respondents had concerns about safety with chiropractic treatment. In some cases, this concern arose only recently despite long-term, uneventful use of chiropractic treatment. This may be related to more recent public emphasis on the rare occurrences of stroke that have been associated with chiropractic manipulation. Although none of the participants had experienced a severe adverse effect with chiropractic treatment, and the evidence on this topic is still being debated [36-38], these patients noted changes in their personal health practices because of contextual beliefs. The Behavioral Model of Health Services emphasizes that the external environment influences health behaviour.

About 1/4 of participants mentioned the financial dynamics of treatment. Financial considerations can be considered at a contextual level (health policy and financing) or at an individual level as contributors to health care utilization. The concern most commonly cited was a lack of funding for treatments they wished to pursue [35]. Again, it was noteworthy that few participants were concerned whether there was evidence for these interventions. This shows some concordance with the experience of family physicians about patients' lack of insight into cost effectiveness [13, 14]. It would appear that greater incorporation of evidence-based decision-making between patients and health care providers might be needed, but will be challenged by differences in beliefs and preferences.

Financial considerations can also be a concern if patients believe that their provider is motivated by financial reimbursement. This was less commonly mentioned by study participants, but a concern for some. There are substantial variations in providers and how they are funded in the provision of health care services for neck pain. Providers may need to be aware of clarity in defining how they are funded and its independence from care as part of informed consent to facilitate a better therapeutic relationship.

Patients expressed needs for diverse types of information and a lack of satisfaction with what they were currently provided. In a study of information-seeking experiences of patients with fibromyalgia, we found that patients experienced a similar frustration with health care providers not being able to provide accurate and useful information about their health problem [39, 40]. Patients did express concerns for quality information similar to that reported in a previous qualitative study of neck and back pain [23]. Participants in the current study indicated a need for practical information on how to cope with their neck pain, and effectively implement their exercise and activity programs. Clinicians may need more awareness about how they can find open-access, high-quality evidence resources to 
recommend to patients that address common musculoskeletal symptoms to meet this need.

The current study has limitations that should be considered. Qualitative research is not inherently generalizable, nor does it confirm hypotheses. Rather, it is an effective means of generating hypotheses. The results of this study, while theoretically sound, should be replicated or supported through quantitative means before we would suggest generalizability. Only 2 English speaking countries with similar health care system were sampled. We cannot generalize these findings to non-English speaking countries or those with different health delivery systems. Further, qualitative research paradigms are collected and interpreted through the lens of the observer (researcher), and, as such, the data and the researcher cannot be separated. It is possible that other researchers looking at the same data would identify themes that are different from those that we have identified. We attempted to address trustworthiness of the results through two independent researchers and a member-checking exercise. Nevertheless, the experiences of the researchers, each of whom have both clinical and academic experience in the field of neck pain, will have likely influenced the results. While this type of reflection is recognized and embraced in qualitative research, it warrants caution when attempting to apply these results to the broader context of health or policy decisions.

This study provides insight into the complexity of defining effective health care services for patients with neck pain. There is clearly no one-size fits all solution, no sure things or optimal professional group. Rather, patients struggle to navigate the variable health services and providers that are available to them and to determine which will meet their needs - often through a trial and error approach. As such, they remain open to unproven, even controversial treatment options. Research evidence was not a key ingredient in patient decision-making about accessing health services. In combination, these factors contribute to financial costs that burden both the individual and society at large. Better understanding of contextual, individual and provider determinants of health care use and effectiveness is needed to complement evidence around the effect of specific treatments.

\section{CONFLICT OF INTEREST}

The authors confirm that this article content has no conflict of interest.

\section{ACKNOWLEDGEMENTS}

ICON is a multi-disciplinary collaborative group that includes scientist-authors (listed below) and support staff (Margaret Lomotan) that conduct knowledge synthesis and translation aimed at reducing the burden of neck pain.

The ICON authors provided direction of the project; input into the interview questions and review of the findings/manuscript; and include (in alphabetical order): Gert Bronfort, Norm Buckley, Lisa Carlesso, Linda Carroll, Pierre Côté, Jeanette Ezzo, Paulo Ferreira, Tim Flynn

Charlie Goldsmith, Anita Gross, Ted Haines, Jan Hartvigsen, Wayne Hing, Gwendolyn Jull, Faith Kaplan, Ron Kaplan, Helge Kasch, Justin Kenardy, Per Kjær, Janet Lowcock, Joy MacDermid, Jordan Miller, Margareta Nordin,
Paul Peloso, Jan Pool, Duncan Reid, Sidney Rubinstein, Lina Santaguida, Anne Söderlund, Natalie Spearing, Michele Sterling, Grace Szeto, Robert Teasell, Arianne Verhagen, David M. Walton, Marc White.

This work was supported by Canadian Institutes of Health Research (CIHR) grant(s) FRN: KRS-102084

The authors wish to thank Liora Bliumkin, Janice Cheung, and Margaret Lomotan for assistance with the study.

\section{REFERENCES}

[1] Picavet HS, Schouten JS. Musculoskeletal pain in the Netherlands: prevalences, consequences and risk groups, the DMC(3)-study. Pain 2003; 102(1-2): 167-78.

[2] Croft PR, Lewis M, Papageorgiou AC, et al. Risk factors for neck pain: a longitudinal study in the general population. Pain 2001; 93(3): 317-25

[3] Cote P, Kristman V, Vidmar M, et al. The prevalence and incidence of work absenteeism involving neck pain: a cohort of Ontario lost-time claimants. Spine (Phila Pa 1976 ) 2008; 33(4 Suppl): S192-8.

[4] Hogg-Johnson S, van der Velde G, Carroll LJ, et al. The burden and determinants of neck pain in the general population: results of the Bone and Joint Decade 2000-2010 Task Force on Neck Pain and Its Associated Disorders. Spine 2008; 33(4 Suppl): S39-51.

[5] Holm LW, Carroll LJ, Cassidy JD, et al. The burden and determinants of neck pain in whiplash-associated disorders after traffic collisions: results of the Bone and Joint Decade 2000-2010 Task Force on Neck Pain and Its Associated Disorders. Spine 2008; 33(4 Suppl): S52-9.

[6] Miettinen T, Lindgren KA, Airaksinen O, Leino E. Whiplash injuries in Finland: a prospective 1-year follow-up study. Clin Exp Rheumatol 2002; 20(3): 399-402.

[7] Cote P, van der Velde G, Cassidy JD, et al. The burden and determinants of neck pain in workers: results of the Bone and Joint Decade 2000-2010 Task Force on Neck Pain and Its Associated Disorders. Spine 2008; 33(Suppl 4): S60-74.

[8] Schmitt KU, Walz F, Vetter D, Muser M. Whiplash injury: cases with a long period of sick leave need biomechanical assessment. Eur Spine J 2003; 12(3): 247-54.

[9] Webb R, Brammah T, Lunt M, Urwin M, Allison T, Symmons D. Prevalence and predictors of intense, chronic, and disabling neck and back pain in the UK general population. Spine (Phila $\mathrm{Pa} 1976$ ) 2003; 28(11): 1195-202.

[10] Borghouts JA, Koes BW, Vondeling H, Bouter LM. Cost-of-illness of neck pain in The Netherlands in 1996. Pain 1999; 80(3): 629-36.

[11] Chevan J, Riddle DL. Factors associated with care seeking from physicians, physical therapists, or chiropractors by persons with spinal pain: a population-based study. J Orthop Sports Phys Ther 2011; 41(7): 467-76.

[12] Driessen MT, Lin CW, van Tulder MW. Cost-effectiveness of conservative treatments for neck pain: a systematic review on economic evaluations. Eur Spine J 2012; 21(8): 1441-50.

[13] Escolar-Reina P, Medina-Mirapeix F, Gascon Canovas JJ, et al. How do care-provider and home exercise program characteristics affect patient adherence in chronic neck and back pain: a qualitative study. BMC Health Serv Res 2010; 10: 60.

[14] Wermeling M, Scherer M, Himmel W. GPs' experiences of managing non-specific neck pain--a qualitative study. Fam Pract 2011;28(3): 300-6.

[15] Russell G, Nicol P. 'I've broken my neck or something!' The general practice experience of whiplash. Fam Pract 2009; 26(2): 115-20.

[16] Scherer M, Schaefer H, Blozik E, Chenot JF, Himmel W. The experience and management of neck pain in general practice: the patients' perspective. Eur Spine J 2010; 19(6): 963-71.

[17] Sweet L. Telephone interviewing: is it compatible with interpretive phenomenological research? Contemp Nurse 2002; 12(1): 58-63.

[18] Hycner RH. Some guidelines for the phenomenological analysis of interview data. Hum Stud 1985; 8: 279-303.

[19] Creswell JW. Qualitative inquiry and research design. Choosing among five approaches. $2^{\text {nd }}$ ed. London: Sage Publications 2007. 
[20] Goldsmith CH, Gross AR, MacDermid J, Santaguida PL, Miller J. What does the evidence tell us about design of future treatment trials for whiplash-associated disorders? Spine (Phila Pa 1976 ) 2011; 36(25 Suppl): S292-302.

[21] Michelle C, Ray F, Andrew H, et al. Framework for design and evaluation of complex interventions to improve health. BMJ 2000; 321(7262): 694-6.

[22] Fjellman-Wiklund A, Stenlund T, Steinholtz K, Ahlgren C. Take charge: Patients' experiences during participation in a rehabilitation programme for burnout. J Rehabil Med 2010; 42(5): 475-81.

[23] Stenberg G, Fjellman-Wiklund A, Ahlgren C. "Getting confirmation": gender in expectations and experiences of healthcare for neck or back patients. J Rehabil Med 2012; 44(2): 163-71.

[24] Hall AM, Ferreira PH, Maher CG, Latimer J, Ferreira ML. The influence of the therapist-patient relationship on treatment outcome in physical rehabilitation: a systematic review. Phys Ther 2010; 90(8): 1099-110.

[25] Krohne K, Ihlebaek C. Maintaining a balance: a focus group study on living and coping with chronic whiplash-associated disorder. BMC Musculoskelet Disord 2010; 11: 158 .

[26] Thom DH, Campbell B. Patient-physician trust: an exploratory study. J Fam Pract 1997; 44(2): 169-76.

[27] Thom DH. Physician behaviors that predict patient trust. J Fam Pract 2001; 50(4): 323-8

[28] Rhodes LA, McPhillips-Tangum CA, Markham C, Klenk R. The power of the visible: the meaning of diagnostic tests in chronic back pain. Soc Sci Med 1999; 48(9): 1189-203.

[29] Andersen RM. Revisiting the behavioral model and access to medical care: does it matter? J Health Soc Behav 1995; 36(1): 1-10.

[30] Aday LA, Andersen R. A framework for the study of access to medical care. Health Serv Res 1974; 9(3): 208-20.
[31] Goode AP, Freburger J, Carey T. Prevalence, practice patterns, and evidence for chronic neck pain. Arthritis Care Res (Hoboken) 2010; 62(11): 1594-601.

[32] Cote P, Clarke J, Deguire S, Frank JW, Yassi A. Chiropractors and return to-work: The experiences of three Canadian focus groups. J Manipulative Physiol Ther 2001; 24(5): 309-16.

[33] Jeffrey JE, Foster NE. A qualitative investigation of physical therapists' experiences and feelings of managing patients with nonspecific low back pain. Phys Ther 2012; 92(2): 266-78.

[34] Gurden M, Morelli M, Sharp G, Baker K, Betts N, Bolton J. Evaluation of a general practitioner referral service for manual treatment of back and neck pain. Prim Health Care Res Dev 2012, 13(3): 204-10.

[35] Bexreh T, Law MB, Taubin T, Rigkin DE, Wilson IB. Challenges to physician-patient communication about medication use: a window into the skeptical patient's world. Patient Prefer Adherence 2012; 6: 11-8.

[36] Albuquerque FC, Hu YC, Dashti SR, et al. Craniocervical arterial dissections as sequelae of chiropractic manipulation: patterns of injury and management. J Neurosurg 2011; 115(6): 1197-205.

[37] Gouveia LO, Castanho P, Ferreira JJ. Safety of chiropractic interventions: a systematic review. Spine (Phila Pa 1976) 2009 34(11): E405-13.

[38] Ernst E. Manipulation of the cervical spine: a systematic review of case reports of serious adverse events, 1995-2001. Med J Aust 2002; 176(8): 376-80.

[39] Daraz L, MacDermid JC, Shaw L, Wilkins S, Gibson J. Experiences of women living with fibromyalgia: an exploratory study of their information needs and preferences. Rhematol Rep 2012; 3(e15):56-60.

[40] Daraz L, Mac Dermid JC, Wilkins S, Gibson J, Shaw L. Information preferences of people living with fibromyalgia - a survey of their information needs and preferences. Rheumatol Rep 2011; 3(e7):26-33

(C) MacDermid et al.; Licensee Bentham Open.

This is an open access article licensed under the terms of the Creative Commons Attribution Non-Commercial License (http://creativecommons.org/licenses/by-nc/3.0/) which permits unrestricted, non-commercial use, distribution and reproduction in any medium, provided the work is properly cited. 